\title{
ON THE EMBEDDING OF LEFT-SYMMETRIC ALGEBRAS INTO DIFFERENTIAL PERM-ALGEBRAS
}

\author{
P. S. KOLESNIKOV AND B. K. SARTAYEV
}

\begin{abstract}
Given an associative algebra satisfying the left commutativity identity $a b c=b a c$ (Perm-algebra) with a derivation $d$, the new operation $a \circ b=a d(b)$ is left-symmetric (pre-Lie). We derive necessary and sufficient conditions for a left-symmetric algebra to be embeddable into a differential Perm-algebra.
\end{abstract}

\section{INTRODUCTION}

The class of left-symmetric algebras (also known as pre-Lie algebras) initially appeared in deformation theory and geometry ([7], [13], [19]). By definition, a left-symmetric algebra is a linear space with one bilinear multiplication satisfying the identity

$$
(a, b, c)=(b, a, c),
$$

where $(a, b, c)=(a b) c-a(b c)$ is the associator.

If, in addition, the right commutativity holds, i.e.,

$$
(a b) c=(a c) b,
$$

then such a system is known as a Novikov algebra. Novikov algebras appeared in formal variational calculus [6] and, independently, as a tool for studying Poisson brackets of hydrodinamic type [2]. The structure theory of Novikov algebras is well-developed, see, e.g., [14 and references therein.

For example, if $A$ is an associative and commutative algebra with a derivation $d$ then the same space $A$ relative to the new product

$$
a \circ b=a d(b), \quad a, b \in A,
$$

is a Novikov algebra. As shown in [3], this example is generic: every Novikov algebra embeds into a differential commutative algebra. An alternative way to prove the latter is to apply the following observation: the free Novikov algebra $\operatorname{Nov}\langle X\rangle$ generated by a set $X$ embeds into the algebra $\operatorname{ComDer}\langle X, d\rangle$ of differential polynomials in $X$ [5], and it is easy to show [12] that every homomorphic image of $\operatorname{Nov}\langle X\rangle$ also embeds into a commutative differential algebra.

This work was supported by the Program of fundamental scientific researches of the Siberian Branch of Russian Academy of Sciences, I.1.1, project 0314-2019-0001. 
In this paper, we study the class of left-symmetric algebras obtained from non-commutative differential algebras by means of the operation (1.1). Namely, if $A$ is an associative algebra satisfying the identity of left commutativity $a b c=b a c$ (i.e., $A$ is a Perm-algebra) then for every derivation $d$ on $A$ the operation (1.1) turns $A$ into a left-symmetric algebra $A^{(d)}$.

Let us call a left-symmetric algebra special if it can be embedded into a differential Perm-algebra via (1.1). Although Perm-algebras are very close to commutative ones, the description of the class of special left-symmetric algebras differs from that of Novikov algebras. The most important difference is that it is not a variety: a homomorphic image of a special left-symmetric algebra may not be special. We show the class of special left-symmetric algebras to be a quasi-variety. It is easy to see that every special left-symmetric algebra satisfies $((a x) y) b=((a y) x) b$ and $(x, y a, b)=(y, x a, b)$. The corresponding variety of $S L S$-algebras contains the class of special left-symmetric algebras and we find explicitly the set of quasi-identities defining special algebras within the SLS-algebras. Finally, we prove that the class of SLS-algebras is the smallest variety containing special left-symmetric algebras. The latter follows from the observation that the free SLS-algebra is special.

We believe that the same approach as developed in this paper will be useful for finding the complete list of special identities for Gelfand-Dorfman algebras. In that case (see [12]) the class of special algebras does form a variety, and it is known that there are two idependent identities of degree 4.

Throughout the paper, $\mathbb{k}$ is an arbitrary base field. We will use the following notations. If Var is a variety of algebras then $\operatorname{Var}\langle X\rangle$ stands for the free algebra in Var generated by a set $X$. We will use the same symbol Var to denote the operad goverining the variety Var.

For example, if Com denotes the variety of associative and commutative algebras then $\operatorname{Com}\langle X\rangle=\mathbb{k}[X]$ is the ordinary polynomial algebra. By LSym we denote the variety of left-symmetric algebras, Nov $\subset$ LSym stands for Novikov algebras, etc.

The class of pairs $(A, d), A \in \operatorname{Var}, d$ is a derivation on $A$ is also a variety denoted VarDer. The free algebra in VarDer generated by a set $X$ is denoted $\operatorname{VarDer}\langle X, d\rangle$. Here we include $d$ into the notation for convenience. As a Var-algebra, $\operatorname{VarDer}\langle X, d\rangle$ is isomorphic to $\operatorname{Var}\left\langle X^{(\omega)}\right\rangle$, where

$$
X^{(\omega)}=X \cup X^{\prime} \cup X^{\prime \prime} \cup \cdots \cup X^{(n)} \cup \ldots,
$$

$X^{(n)}=\left\{x^{(n)} \mid x \in X\right\}, n \geq 0$, are disjoint copies of $X$. The derivation $d$ acts on the generators of $\operatorname{Var}\left\langle X^{(\omega)}\right\rangle$ as $d\left(x^{(n)}\right)=x^{(n+1)}$.

The interest to the study of differential Perm-algebras has one more motivation. The operad Perm plays a specific role in the theory of dialgebras [15] and their operads (called replicated operads) [8], [17]: given an operad Var, the Manin white product of operads PermoVar is exactly the operad governing 
the class diVar of Var-dialgebras. Note that for Perm the Manin white product coincides with the Hadamard product of operads.

On the other hand, the operad Nov has its own distinguished role in the combinatorics of derivations on non-associative algebras [11]: given a binary operad Var, the Manin white product Nov o Var is the operad governing the class of derived Var-algebras. The latter are obtained from Var-algebras with a derivation $d$ relative to the new operations

$$
a \prec b=a d(b), \quad a \succ b=d(a) b
$$

(for each of binary products in Var, if there are more than one operation).

In the problem we consider in this paper, Perm and Nov meet each other since we have to study differential Perm-algebras. This is why the theory of dialgebras (especially, Novikov dialgebras) provides us with effective tools for studying speciality of left-symmetric algebras.

The paper is organized as follows.

In Section 2 we recall the definition of a Var-dialgebra and prove an analogue of the embedding theorem from [3] for Novikov dialgebras. This is done in a routine way by means of the general methods described in [8] (see also [9]). Since every Novikov dialgebra is in particular a left-symmetric algebra, we obtain that an embedding of a left-symmetric algebra into a differential Permalgebra is equivalent to the embedding into a Novikov dialgebra.

Section 3 is devoted to the study of the forgetful functor from diLSym to LSym. It turns out that the left adjoint functor has a very natural description: given an algebra $A \in \mathrm{LSym}$, we may define the structure of its universal enveloping left-symmeric dialgebra on the tensor algebra $T(A)$.

In Section 4, we prove that the free SLS-algebra is special. Namely, the subalgebra of PermDer $\langle X, d\rangle$ generated by the set $X$ relative to the operation $a \circ b=a d(b)$ is isomorphic to the free left-symmetric algebra satisfying two identities of SLS-algebras mentioned above. In contrast to [5], we do not find the monomial basis of $\operatorname{SLS}\langle X\rangle$, but still we can describe its linear basis explicitly as a subset of $\operatorname{PermDer}\langle X, d\rangle$.

Finally, in Section 5 we derive the necessary conditions of speciality for an SLS-algebra in the form of quasi-identities. Then we prove that these conditions are also sufficient. Given an SLS-algebra $A$, we construct an appropriate quotient of its universal enveloping diLSym-algebra constructed in Section 3 to get a Novikov dialgebra envelope of $A$. The results of Section 2 then lead us to the desired conclusion.

It is easy to construct examples of non-special SLS-algebras, however, the free SLS-algebra is special (Section 4). Hence, the class of special left-symmetric algebras does not form a variety. 


\section{Novikov Dialgebras and Differential Perm-Algebras}

Let Perm denote the class of associative algebras satisfying the identity

$$
x y z-y x z=0 .
$$

The operad governing the variety of such algebras is also denoted Perm. It is clear (see [4]) that $\operatorname{dim} \operatorname{Perm}(n)=n$, and the composition rule is easy to describe [10].

Given a binary quadratic operad $\mathcal{O}$, the Hadamard product of operads $\mathcal{O} \otimes$ Perm coincides with the Manin white product [16], and the class of algebras governed by $\mathcal{O} \otimes$ Perm is known as the variety of di $\mathcal{O}$-algebras (replicated $\mathcal{O}$-algebras [17, or $\mathcal{O}$-dialgebras [10]). In particular, if Com and Lie denote the operads governing commutative and Lie algebras then diCom and diLie are isomorphic to Perm and Leib, respectively, where Leib is the operad of Leibniz algebras.

If $\operatorname{dim} O(2)=2$ (one binary product with no symmetry) then the operad di $\mathcal{O}$ is generated by two operations $\vdash$ and $\dashv$. The defining relations of $\operatorname{di} \mathcal{O}$ are easy to derive from those of $\mathcal{O}$ [10]. Namely, every di $\mathcal{O}$-algebra satisfies so-called 0-identities

$$
\begin{aligned}
& \left(x_{1} \dashv x_{2}\right) \vdash x_{3}=\left(x_{1} \vdash x_{2}\right) \vdash x_{3}, \\
& x_{1} \dashv\left(x_{2} \vdash x_{3}\right)=x_{1} \dashv\left(x_{2} \dashv x_{3}\right) .
\end{aligned}
$$

Moreover, for every multi-linear defining identity $f\left(x_{1}, \ldots, x_{n}\right)=0$ of $\mathcal{O}$ and for every $i=1, \ldots, n$ one should claim

$$
f_{i}\left(x_{1}, \ldots, x_{n}\right)=f\left(x_{1}, \ldots, \dot{x}_{i}, \ldots, x_{n}\right)=0
$$

to hold on di $\mathcal{O}$. Here $f\left(x_{1}, \ldots, \dot{x}_{i}, \ldots, x_{n}\right)$ is derived from $f$ in the following way: in each nonassociative monomial $\left(x_{j_{1}} \ldots x_{j_{n}}\right)$ the initial binary product is replaced with the products $\vdash$ and $\dashv$ so that the horizontal dashes point to the fixed variable $x_{i}$.

Example 1. Let Nov stand for the variety of Novikov algebras as well as for the corresponding operad. Then diNov is defined by (2.1) together with

$$
\begin{gathered}
\left(x_{1} \dashv x_{2}\right) \dashv x_{3}-x_{1} \dashv\left(x_{2} \dashv x_{3}\right)=\left(x_{2} \vdash x_{1}\right) \dashv x_{3}-x_{2} \vdash\left(x_{1} \dashv x_{3}\right), \\
\left(x_{1} \vdash x_{2}\right) \vdash x_{3}-x_{1} \vdash\left(x_{2} \vdash x_{3}\right)=\left(x_{2} \vdash x_{1}\right) \vdash x_{3}-x_{2} \vdash\left(x_{1} \vdash x_{3}\right), \\
\left(x_{1} \dashv x_{2}\right) \dashv x_{3}=\left(x_{1} \dashv x_{3}\right) \dashv x_{2}, \\
\left(x_{1} \vdash x_{2}\right) \dashv x_{3}=\left(x_{1} \vdash x_{3}\right) \vdash x_{2} .
\end{gathered}
$$

A linear space $N$ equipped with bilinear operations $\vdash$ and $\dashv$ is a Novikov dialgebra if and only if (2.1)-(2.3) hold for all $x_{1}, x_{2}, x_{3} \in N$.

Obviously, every Perm-algebra (diCom-algebra) with a derivation $d$ turns into a Novikov dialgebra (diNov-algebra) relative to the new operations $x \vdash$ 
$y=x d(y), x \dashv y=d(y) x$. The embedding statement may be derived by means of the general construction from [8] that allows us to reduce the problem on a Novikov dialgebra to an "ordinary" Novikov algebra by means of conformal algebras (pseudo-algebras). One may refer to [1] for details on pseudo-algebras, but for our puproses the only essential instance is that every pseudo-algebra may be turned into a dialgebra [10].

Theorem 1. Every Novikov dialgebra $(N, \vdash,-)$ may be embedded into a differential Perm-algebra in such a way that

$$
x \vdash y=x d(y), \quad x \dashv y=d(y) x
$$

for $x, y \in N$.

Proof. If $N$ is a Novikov dialgebra with operations $\vdash$ and $\dashv$ then

$$
N_{0}=\operatorname{span}\{a \vdash b-a \dashv b \mid a, b \in N\}
$$

is an ideal in $N$ and $\bar{N}=N / N_{0}$ is a Novikov algebra. The space $N$ is a Novikov bimodule over $\bar{N}$ relative to the action

$$
\bar{a} \circ b=a \vdash b, \quad a \circ \bar{b}=a \dashv b,
$$

for $a, b \in N$. The corresponding split null extension $\hat{N}=\bar{N} \ltimes N$ is a Novikov algebra [18] in which $N \circ N=0$.

Let $H$ be a cocommutative bialgebra with a primitive element $T$ (e.g., $H=$ $\mathbb{k}[T])$. Consider the current pseudo-algebra $\operatorname{Cur} \hat{N}=H \otimes \hat{N}$. Then $\operatorname{Cur} \hat{N}$ is a Novikov dialgebra relative to the operations

$$
(f \otimes x) \vdash(g \otimes y)=\varepsilon(f) g \otimes x \circ y, \quad(f \otimes x) \dashv(g \otimes y)=\varepsilon(g) f \otimes x \circ y
$$

for $x, y \in \hat{N}, f, g \in H$, where $\varepsilon$ is the counit in $H$.

The dialgebra $N$ embeds into $\operatorname{Cur} \hat{N}$ via

$$
a \mapsto \hat{a}=1 \otimes \bar{a}+T \otimes a, \quad a \in N
$$

Let $(B, \cdot d)$ be a commutative differential algebra which contains $\hat{N}$ in such a way that

$$
x \cdot d(y)=x \circ y, \quad x, y \in \hat{N} .
$$

Then $C=\operatorname{Cur} B$ may be considered as a commutative dialgebra (i.e., Permalgebra) and it is easy to check that $\hat{d}=\mathrm{id} \otimes d$ is a derivation of $C$. Thus we have

$$
N \subset \operatorname{Cur} \hat{N} \subset \operatorname{Cur} B=C,
$$

and it is easy to check that the operations $\vdash, \dashv$ on $N$ are related with the operations $\cdot, \hat{d}$ on $C$ in the desired way. Indeed, if $a, b \in N, \hat{a}, \hat{b} \in \operatorname{Cur} \hat{N}$ then 
the product of $\hat{d}(\hat{b})$ and $\hat{a}$ in the Perm-algebra $C$ may be calculated as follows:

$$
\begin{array}{r}
\hat{d}(\hat{b}) \hat{a}=(1 \otimes d(\bar{b})+T \otimes d(b))(1 \otimes \bar{a}+T \otimes a)=1 \otimes d(\bar{b}) \cdot \bar{a}+T \otimes d(\bar{b}) \cdot a) \\
=1 \otimes(\bar{a} \circ \bar{b})+T \otimes(a \circ \bar{b})=1 \otimes \overline{a \dashv b}+T \otimes(a \dashv b)=\widehat{a \dashv b} .
\end{array}
$$

In a similar way, $\widehat{a \vdash b}=\hat{a} \hat{d}(\hat{b})$ in $C$ for all $a, b \in N$.

Corollary 1. A left-symmetric algebra $(A, \circ)$ embeds into a differential Perm algebra in such a way that $a \circ b=a d(b)$ for $a, b \in A$ if and only if $(A, \circ)$ embeds into a Novikov dialgebra in such a way that $a \vdash b=a \circ b$ for $a, b \in A$.

\section{UNIVERSAL ENVELOPING LEFT-SYMMETRIC DIALGEBRAS}

According to the general rule of constructing defining identities of dialgebras, every left-symmetric dialgebra $(A, \vdash, \dashv)$ is a left-symmetric algebra relative to the operation $\vdash$. Thus we have a forgetful functor diLSym $\rightarrow$ LSym. In this subsection, we explicitly construct its left adjoint functor $\mathcal{D}:$ LSym $\rightarrow$ diLSym which will be applied in the sequel.

Let $(A, \circ)$ be a left-symmetric algebra. Consider the (associative) tensor algebra $T(A)$ of the space $A$ (without the identity):

$$
T(A)=A \oplus A^{\otimes 2} \oplus A^{\otimes 3} \oplus \ldots
$$

We will write $a_{1} \ldots a_{n}$ for the element $a_{1} \otimes \cdots \otimes a_{n} \in A^{\otimes n}$ for brevity.

Define the map $M: T(A) \rightarrow A$ as follows:

$$
M\left(a_{1} \ldots a_{n}\right)=\left(\left(\ldots\left(\left(a_{1} \circ a_{2}\right) \circ a_{3}\right) \circ \ldots\right) \circ a_{n}\right), \quad a_{i} \in A .
$$

Let us define a binary operation $\vdash$ on the space $T(A)$ by induction on the length of the second factor:

$$
\begin{gathered}
u \vdash a=M(u) \circ a, \quad u \in T(A), a \in A, \\
u \vdash(w a)=(u \vdash w) a+w(M(u) \circ a)-w M(u) a,
\end{gathered}
$$

for $u, w \in T(A), a \in A$. Finally, define a binary operation $\dashv$ on $T(A)$ as

$$
u \dashv w=u M(w), \quad u, w \in T(A) .
$$

Lemma 1. For every $u, w \in T(A)$ we have

$$
\begin{gathered}
u \vdash w=M(u) \vdash w, \quad u \dashv w=u \vdash M(w), \\
M(u \vdash w)=M(u \dashv w)=M(u) \circ M(w) .
\end{gathered}
$$


Proof. The desired equalities are obvious for the operation $\dashv$, as well as for $w=a \in A$. Proceed by induction on the length of $w$ :

$$
\begin{array}{r}
M(u \vdash w a)=M(u \vdash w) \circ a+M(w) \circ(M(u) \circ a)-(M(w) \circ M(u)) \circ a \\
=(M(u) \circ M(w)) \circ a-(M(w), M(u), a)=M(u) \circ(M(w) \circ a) \\
=M(u) \circ M(w a)
\end{array}
$$

by the left symmetry.

Proposition 1. For every left-symmetric algebra $(A, \circ)$, the space $T(A)$ equipped with the operations (3.1), (3.2) is a left-symmetric dialgebra.

Proof. It is enough to check that (2.1) and (2.2) hold on $(T(A), \vdash, \dashv)$.

Let us start with (2.1). Consider $u, v, w \in T(A)$ and note that

$$
\begin{aligned}
& (u \vdash v) \vdash w=M(u \vdash v) \vdash w \\
& \quad=(M(u) \circ M(v)) \vdash w=M(u \dashv v) \vdash w=(u \dashv v) \vdash w
\end{aligned}
$$

by Lemma 1. The remaining 0-identity holds by similar reasons.

Next, proceed to (2.2). Let us state the calculation for the more complicated case: show that the "right" associator

$$
(u \vdash v) \vdash w-u \vdash(v \vdash w)
$$

is symmetric relative to the exchange of $u$ and $v$. Indeed, for $w=c \in A$ it is enough to apply Lemma 1 and the left symmetry of $A$. For the general case, apply induction on the length of $w$. Since we may replace $u$ and $v$ with $M(u)$ and $M(v)$ by Lemma 1, consider $u=a, v=b(a, b \in A)$ :

$$
\begin{aligned}
(a \vdash b) \vdash w c-a \vdash(b \vdash w c) & \\
= & ((a \circ b) \vdash w) c+w((a \circ b) \circ c)-w(a \circ b) c \\
& \quad-a \vdash((b \vdash w) c+w(b \circ c)-w b c) \\
= & ((a \circ b) \vdash w) c+w((a \circ b) \circ c)-w(a \circ b) c \\
- & (a \vdash(b \vdash w)) c-(b \vdash w)(a \circ c)+(b \vdash w) a c \\
- & (a \vdash w)(b \circ c)-w(a \circ(b \circ c))+w a(b \circ c) \\
& +(a \vdash w b) c+w b(a \circ c)-w b a c .
\end{aligned}
$$

Let us remove those summands that already form symmetric expressions (relative to the exchange of $a$ and $b)$ and expand the last $(a \vdash w b)$ :

$$
\begin{aligned}
-w(a \circ b) c-(b \vdash w)(a \circ c)+(b \vdash w) a c-(a \vdash w)(b \circ c)+w a(b \circ c) \\
+(a \vdash w) b c+w(a \circ b) c-w a b c+w b(a \circ c)-w b a c \\
=(a \vdash w) b c+(b \vdash w) a c-(a \vdash w)(b \circ c)-(b \vdash w)(a \circ c) \\
+w a(b \circ c)+w b(a \circ c)-w b a c-w a b c .
\end{aligned}
$$


The expression obtained has the desired symmetry.

The remaining relation from $(2.2)$ can be proved in a similar but simpler way.

Denote the left-symmetric dialgebra $(T(A), \vdash,-)$ by $\mathcal{D}(A)$. The injection $\iota: A \rightarrow \mathcal{D}(A), \iota(a)=a$, is a homomorphism from $A$ to $\mathcal{D}(A)^{\circ}=(\mathcal{D}(A), \vdash)$ by the definition.

Proposition 2. Let $(A, \circ)$ be a left-symmetric algebra. Then for every leftsymmetric dialgebra $B$ and for every homomorphism $\tau: A \rightarrow B^{\circ}$ there exists unique homomorphism of dialgebras $\varphi: \mathcal{D}(A) \rightarrow B$ such that $\varphi \iota=\tau$.

Proof. It is enough to check that the map

$$
\varphi\left(a_{1} a_{2} \ldots a_{n-1} a_{n}\right)=\left(\ldots\left(\left(x_{1} \dashv x_{2}\right) \dashv \ldots \dashv x_{n-1}\right) \dashv x_{n}\right), \quad x_{i}=\tau\left(a_{i}\right),
$$

is a homomorphism of dialgebras preserving $\vdash$ and $\dashv$. The latter follows from the following observation: for all $w \in T(A), x \in B$ we have

$$
\varphi(w) \vdash x=\tau(M(w)) \vdash x, \quad x \dashv \varphi(w)=x \dashv \tau(M(w))
$$

by (2.1). It remains to prove

$$
\varphi(a \vdash w)=\tau(a) \vdash \varphi(w), \quad a \in A,
$$

by induction on the length of $w \in T(A)$, and apply Lemma 1 .

Thus $\mathcal{D}(A)$ is the universal enveloping left-symmetric dialgebra of of a leftsymmetric algebra $A$. As we can see, every $A$ embeds into its universal dialgebra envelope which is just $T(A)$ as a linear space. The functor $\mathcal{D}(\cdot)$ is left adjoint to the forgetful functor diLSym $\rightarrow$ LSym which is induced by the morphism of operads $x_{1} \circ x_{2} \mapsto x_{1} \vdash x_{2}$.

Note that the same sort of functor acts on diNov: every Novikov dialgebra is in particular a left-symmetric algebra relative to the operation $\vdash$. So one may construct a universal enveloping Novikov dialgebra for a left-symmetric algebra $A$, but the problem is to distinguish those algebras that are embedded into such envelopes. We will completely resolve this problem in Section 5.

\section{A linear basis of the FREe SLS-ALGEBra}

Let $P$ be a Perm-algebra with a derivation $d$. As above, denote $d(x)$ by $x^{\prime}$ for $x \in P$. A new binary operation

$$
x \circ y=x y^{\prime}, \quad x, y \in P,
$$

turns $P$ into a left-symmetric algebra. Moreover, the following identities hold on $P$ :

$$
\begin{gathered}
((x \circ y) \circ z) \circ u=((x \circ z) \circ y) \circ u, \\
(x, y \circ z, u)=(y, x \circ z, u),
\end{gathered}
$$


where $(a, b, c)$ stands for the associator $(a \circ b) \circ c-a \circ(b \circ c)$.

Definition 1. A left-symmetric algebra $A$ satisfying (4.2) and (4.3) is said to be an SLS-algebra.

Let $X$ be a nonempty set. In this section, we prove that the free SLS-algebra $\mathrm{SLS}\langle X\rangle$ embeds into the free differential Perm-algebra PermDer $\langle X, d\rangle$ relative to the operation (4.1). Namely, define a homomorphism

$$
\tau: \operatorname{SLS}\langle X\rangle \rightarrow \operatorname{PermDer}\langle X, d\rangle
$$

such that

$$
\tau(x)=x, x \in X, \quad \tau(f \circ g)=\tau(f) \tau(g)^{\prime} .
$$

The main purpose of this section is to prove that $\tau$ is injective.

A Novikov algebra is in particular an SLS-algebra. Hence, there exists a homomorphism

$$
\phi: \operatorname{SLS}\langle X\rangle \rightarrow \operatorname{Nov}\langle X\rangle .
$$

Choose a monomial basis $\bar{N}$ in $\operatorname{Nov}\langle X\rangle$ and for every $u \in \bar{N}$ choose its monomial pre-image in SLS $\langle X\rangle$. Denote the set of such pre-images by $N$. There exists a monomial basis $B$ of $\operatorname{SLS}\langle X\rangle$ such that $N \subset B$. Let us choose and fix such a set $B$.

Every non-associative word $u$ in $X$ can be written as

$$
u=L\left(u_{1}, \ldots, u_{k}, x\right):=u_{1} \circ\left(u_{2} \circ\left(\cdots \circ\left(u_{k} \circ x\right) \ldots\right)\right),
$$

where $u_{j}$ are non-associative words, $x \in X$. Let $k$ be the length of $u$. For a linear combination of (4.4), the length is the maximal length of its summands.

Lemma 2. Every element of $\mathrm{SLS}\langle X\rangle$ may be written as a linear combination of words of the form (4.4) with $u_{j} \in N, x \in X$.

Proof. It follows from (4.2) that if $\phi(a)=\phi(b)$ then $a \circ u=b \circ u$ for all $a, b, u \in \operatorname{SLS}\langle X\rangle$. This observation makes the lemma obvious.

There exist various presentations of an element $f \in \mathrm{SLS}\langle X\rangle$ by linear combinations of (4.4) as in Lemma 2. However, for every nonzero $f$ we may choose a presentation with minimal length. In this way, we obtain a well-defined length function

$$
\ell: \operatorname{SLS}\langle X\rangle \backslash\{0\} \rightarrow \mathbb{Z}_{+}
$$

which depends only on the choice of $B$.

Recall the notion of weight in a free commutative differential algebra [5]. Let $X^{(\omega)}$ stand for the disjoint union $X \cup X^{\prime} \cup X^{\prime \prime} \cup \ldots$, then $\operatorname{ComDer}\langle X, d\rangle$ as a commutative algebra coincides with the polynomial algebra $\operatorname{Com}\left\langle X^{(\omega)}\right\rangle$. Define

$$
\operatorname{wt}\left(x^{(j)}\right)=j-1, \quad x \in X, j \geq 0,
$$

and set $\operatorname{wt}(u v)=\operatorname{wt}(u)+\operatorname{wt}(v)$ for all monomials $u, v \in \operatorname{Com}\left\langle X^{(\omega)}\right\rangle$. 
As shown in [5], the subspace $W$ spanned in $\operatorname{Com}\left\langle X^{(\omega)}\right\rangle$ by all monomials of weight -1 is isomorphic to the free Novikov algebra $\operatorname{Nov}\langle X\rangle$ relative to the map

$$
\operatorname{Nov}\langle X\rangle \rightarrow \operatorname{Com}\left\langle X^{(\omega)}\right\rangle
$$

defined in the same way as $\tau$. We will not distinguish notations for the maps presenting horizontal arrows in the diagram below.

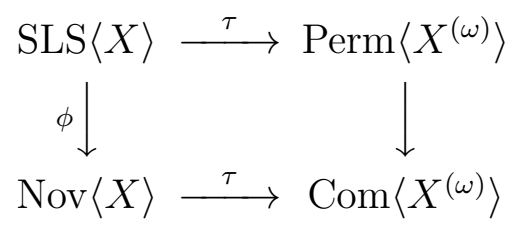

Hence, for every monomial $u \in W$ we may find its unique pre-image $\tau^{-1}(u) \in$ $\operatorname{Nov}\langle X\rangle$, write $\tau^{-1}(u)$ as a linear combination of $N$, and consider the same combination as an element of $\operatorname{SLS}\langle X\rangle$ since $N \subset B$. Thus we obtain a welldefined map $\tau^{-1}: W \rightarrow \mathbb{k} N \subset \mathrm{SLS}\langle X\rangle$ which depends only on the choice of $B$.

If $u \in \operatorname{SLS}\langle X\rangle$ is a monomial of the form (4.4) then

$$
\tau(u)=\tau\left(u_{1}\right) \tau\left(u_{2}\right) \ldots \tau\left(u_{k}\right) x^{(k)}+g_{u} \in \operatorname{Perm}\left\langle X^{(\omega)}\right\rangle
$$

where all monomials in $g_{u}$ end with $x^{(j)}$ for $j<k$.

Recall that a linear basis of $\operatorname{Perm}\left\langle X^{(\omega)}\right\rangle$ consists of all monomials of the form $u x^{(j)}$, where $j \geq 0, x \in X$, and $u$ is from a linear basis of the free associative commutative algebra $\operatorname{Com}\left\langle X^{(\omega)}\right\rangle$ (see, e.g., [4]). In particular, associative words with different last letters are linearly independent in the free Perm-algebra.

Assume $\operatorname{Ker} \tau \neq 0$ in $\operatorname{SLS}\langle X\rangle$. Choose a nonzero element $f \in \operatorname{Ker} \tau$ of minimal length, $\ell(f)=k$. Without loss of generality we may suppose that all monomials in $f$ in a presentation given by Lemma 2 end with the same letter $x \in X$ :

$$
f=\sum_{j} \alpha_{j} L\left(u_{1 j}, u_{2 j}, \ldots, u_{k j}, x\right)+\tilde{f}
$$

where $\ell(\tilde{f})<k$ or $\tilde{f}=0$. Then

$$
\tau(f)=\sum_{j} \alpha_{j} \tau\left(u_{1 j}\right) \ldots \tau\left(u_{k j}\right) x^{(k)}+h
$$

where all monomials in $h$ end with $x^{(j)}$ for $j<k$. Hence,

$$
\sum_{j} \alpha_{j} \tau\left(u_{1 j}\right) \ldots \tau\left(u_{k j}\right)=0
$$

in $\operatorname{ComDer}\langle X, d\rangle=\operatorname{Com}\left\langle X^{(\omega)}\right\rangle$.

The following statement leads us to a contradiction with the choice of $f$. 
Proposition 3. Let

$$
F=\sum_{j} \alpha_{j} w_{1 j} \otimes \cdots \otimes w_{k j} \in W^{\otimes k}, \quad \alpha_{j} \in \mathbb{k},
$$

turn into zero by multiplication, i.e.,

$$
\mu(F):=\sum_{j} \alpha_{j} w_{1 j} \ldots w_{k j}=0 \in \operatorname{Com}\left\langle X^{(\omega)}\right\rangle .
$$

Then $\ell(g)<k$ for

$$
g=\sum_{j} \alpha_{j} L\left(u_{1 j}, u_{2 j}, \ldots, u_{k j}, x\right),
$$

where $u_{i j}=\tau^{-1}\left(w_{i j}\right), x \in X$.

Before we proceed to the proof, let us state two examples to explain the meaning of Proposition 3 ,

(1) $F=w_{1} \otimes w_{2}-w_{2} \otimes w_{1} \in W^{\otimes 2}$ turns into zero by multiplication. Then for $u_{i}=\tau^{-1}\left(w_{i}\right) \in \mathbb{k} N \subset \operatorname{SLS}\langle X\rangle, i=1,2$, we have

$$
u_{1} \circ\left(u_{2} \circ x\right)-u_{2} \circ\left(u_{1} \circ x\right)=\left(u_{1} \circ u_{2}\right) \circ x-\left(u_{2} \circ u_{1}\right) \circ x
$$

by left symmetry. The right-hand side is of length $\leq 1$ which is smaller than $k=2$.

(2) $F=w_{1} \otimes w_{2} w_{3}^{\prime}-w_{2} \otimes w_{1} w_{3}^{\prime} \in W^{\otimes 2}$ turns into zero by multiplication (here $w_{i} \in W$ ). Then for $u_{i}=\tau^{-1}\left(w_{i}\right)$, we have $u_{i} \circ u_{3} \equiv \tau^{-1}\left(w_{i} w_{3}^{\prime}\right)$ $(\bmod \operatorname{Ker} \phi)$ by definition, and thus

$$
\begin{aligned}
& \tau^{-1}\left(w_{1}\right) \circ\left(\tau^{-1}\left(w_{2} w_{3}^{\prime}\right) \circ x\right)-\tau^{-1}\left(w_{2}\right) \circ\left(\tau^{-1}\left(w_{1} w_{3}^{\prime}\right) \circ x\right) \\
= & u_{1} \circ\left(\left(u_{2} \circ u\right) \circ x\right)-u_{2} \circ\left(\left(u_{1} \circ u\right) \circ x\right)=\left(u_{1} \circ\left(u_{2} \circ u\right)\right) \circ x-\left(u_{2} \circ\left(u_{1} \circ u\right)\right) \circ x
\end{aligned}
$$

by (4.3). The right-hand side is of length $\leq 1$.

Proof. Suppose $\mu(F)=0$ for $F \in W^{\otimes k}$. Then $F$ may be presented as a linear combination of tensors of the form

$$
w_{1} \otimes \cdots \otimes w_{j-1} \otimes(a b \otimes c d-c b \otimes a d) \otimes w_{j+1} \otimes \cdots \otimes w_{k},
$$

where $w_{i}, a, b, c, d$ are monomials in $\operatorname{Com}\left\langle X^{(\omega)}\right\rangle$ and all tensor factors are of weight -1 .

It remains to note that

$$
\left(\tau^{-1}(a b), \tau^{-1}(c d), u\right)=\left(\tau^{-1}(c b), \tau^{-1}(a d), u\right)
$$

holds in $\operatorname{SLS}\langle X\rangle$ for all appropriate $a, b, c, d \in \operatorname{Com}\left\langle X^{(\omega)}\right\rangle$ and for all $u \in$ $\operatorname{SLS}\langle X\rangle$. Since $\operatorname{wt}(a b)=\operatorname{wt}(c d)=-1$ and due to left-symmetry of an SLSalgebra, it is enough to consider the case when $\operatorname{wt}(a)=\operatorname{wt}(c)=-1$. 
Let $x=\tau^{-1}(a)$. It follows from the definition of $\tau$ that for every $b$ such that $\mathrm{wt}(b)=0$ the element $\tau^{-1}(a b)$ considered in $\operatorname{Nov}\langle X\rangle$ is a linear combination of elements

$$
R\left(x, u_{1}, \ldots, u_{m}\right)=\left(\ldots\left(\left(x \circ u_{1}\right) \circ u_{2}\right) \circ \ldots\right) \circ u_{m}, \quad u_{i} \in N .
$$

It follows from (4.2) that for every $u \in B$ we may write

$$
\tau^{-1}(a b) \circ u=R\left(x, u_{1}, \ldots, u_{m}\right) \circ u \in \operatorname{SLS}\langle X\rangle
$$

In a similar way, one may present $\tau^{-1}(c d)$ in $\operatorname{Nov}\langle X\rangle$ as $R\left(y, v_{1}, \ldots, v_{l}\right)$. Then

$$
\begin{aligned}
& \left(\tau^{-1}(a b), \tau^{-1}(c d), u\right)=\left(R\left(x, u_{1}, \ldots, u_{m}\right), R\left(y, v_{1}, \ldots, v_{l}\right), u\right), \\
& \left(\tau^{-1}(c b), \tau^{-1}(a d), u\right)=\left(R\left(y, u_{1}, \ldots, u_{m}\right), R\left(x, v_{1}, \ldots, v_{l}\right), u\right) .
\end{aligned}
$$

The right-hand sides of the last two relations are equal due to the identities of SLS-algebras.

Indeed, let us prove the identity

$$
\left(R\left(x, u_{1}, \ldots, u_{m}\right), R\left(y, v_{1}, \ldots, v_{l}\right), u\right)=\left(R\left(y, u_{1}, \ldots, u_{m}\right), R\left(x, v_{1}, \ldots, v_{l}\right), u\right)
$$

by induction on $m$ and $l$.

If $m=l=0$ then (4.6) coincides with left symmetry. Assume $m=0$, $l>0$ and (4.6) is already proved for smaller $l$ 's. Then (4.3) together with left symmetry imply

$$
\begin{aligned}
&\left(x, R\left(y, v_{1}, \ldots, v_{l}\right), u\right)=\left(x, R\left(y, v_{1}, \ldots, v_{l-1}\right)\right.\left.\circ v_{l}, u\right) \\
&=\left(R\left(y, v_{1}, \ldots, v_{l-1}\right), x \circ v_{l}, u\right)=\left(x \circ v_{l}, R\left(y, v_{1}, \ldots, v_{l-1}\right), u\right) \\
&=\left(y, R\left(x \circ v_{l}, v_{1}, \ldots, v_{l-1}\right), u\right)
\end{aligned}
$$

by induction. It remains to note that $R\left(x \circ v_{l}, v_{1}, \ldots, v_{l-1}\right)$ may be replaced here with $R\left(x, v_{1}, \ldots, v_{l-1}, v_{l}\right)$ by (4.2).

For $m>0$, proceed in a similar way:

$$
\begin{gathered}
\left(R\left(x, u_{1}, \ldots, u_{m}\right), R\left(y, v_{1}, \ldots, v_{l}\right), u\right)=\left(y, R\left(R\left(x, u_{1}, \ldots, u_{m}\right), v_{1}, \ldots, v_{l}\right), u\right) \\
=\left(y, R\left(x, u_{1}, \ldots, u_{m}, v_{1}, \ldots, v_{l}\right), u\right)=\left(y, R\left(x, v_{1}, \ldots, v_{l}, u_{1}, \ldots, u_{m}\right), u\right) \\
=\left(y, R\left(R\left(x, v_{1}, \ldots, v_{l}\right), u_{1}, \ldots, u_{m}\right), u\right)=\left(R\left(x, v_{1}, \ldots, v_{l}\right), R\left(y, u_{1}, \ldots, u_{m}\right), u\right) \\
=\left(R\left(y, u_{1}, \ldots, u_{m}\right), R\left(x, v_{1}, \ldots, v_{l}\right), u\right) .
\end{gathered}
$$

As a result,

$$
\begin{aligned}
& L\left(\tau^{-1}\left(w_{1}\right), \ldots, \tau^{-1}\left(w_{j-1}\right), \tau^{-1}(a b), \tau^{-1}(c d), \tau^{-1}\left(w_{j+1}\right), \ldots \tau^{-1}\left(w_{k}\right), x\right) \\
& \quad-L\left(\tau^{-1}\left(w_{1}\right), \ldots, \tau^{-1}\left(w_{j-1}\right), \tau^{-1}(c b), \tau^{-1}(a d), \tau^{-1}\left(w_{j+1}\right), \ldots \tau^{-1}\left(w_{k}\right), x\right) \\
& \equiv L\left(\tau^{-1}\left(w_{1}\right), \ldots, \tau^{-1}\left(w_{j-1}\right),\left(\tau^{-1}(a b), \tau^{-1}(c d), u\right)-\left(\tau^{-1}(c b), \tau^{-1}(a d), u\right)\right)
\end{aligned}
$$


modulo summands of smaller length, where $u=L\left(\tau^{-1}\left(w_{j+1}\right), \ldots \tau^{-1}\left(w_{k}\right), x\right)$. The right-hand side of (4.7) is zero by (4.6).

Summarizing the exposition of the section, we may state

Theorem 2. For a nonempty set $X$, the subalgebra $F$ generated by $X$ in the free differential Perm-algebra PermDer $\langle X, d\rangle$ relative to the operation $f \circ g=$ $f d(g), f, g \in \operatorname{PermDer}\langle X, d\rangle$ is isomorphic to the free SLS-algebra generated by $X$.

Corollary 2. A linear basis of the subalgebra $F$ mentioned in Theorem 2 consists of all monomials $u \in \operatorname{Perm}\left\langle X^{(\omega)}\right\rangle$ of weight -1 such that $u=v x^{(n)}$, $x \in X, n>0$.

The proof is completely analogous to that of [5] for $\operatorname{ComDer}\langle X, d\rangle$.

\section{A CRITERION OF SPECIALity}

Let us say that an SLS-algebra $(A, \circ)$ is nice if for every $x \in A$ there exists a linear map $\mu_{x}: A \circ A \rightarrow A$ such that $\mu_{x}(a \circ b)=(a \circ x) \circ b$ for all $a, b \in A$.

It is easy to see that $(A, \circ)$ is nice if and only if for every $a_{i}, b_{i} \in A(i=$ $1, \ldots, n)$ the equality

$$
\sum_{i} a_{i} \circ b_{i}=0
$$

implies

$$
\sum_{i}\left(a_{i} \circ x\right) \circ b_{i}=0
$$

for all $x \in A$.

Example 2. A Novikov algebra is a nice SLS-algebra, the operator $\mu_{x}$ coincides with the right multiplication by $x$.

Example 3. Let $V$ be a linear space and let $A=T(V) / I$, where $I$ is the ideal spanned by

$$
a_{0} a_{1} \ldots a_{n} a_{n+1}-a_{0} a_{\sigma(1)} \ldots a_{\sigma(n)} a_{n+1}, \quad a_{i} \in V, \sigma \in S_{n} .
$$

Obviously, $A$ is an associative (thus, left-symmetric) algebra satisfying the identities of SLS-algebras. This is not a Novikov algebra, but it is easy to see that if $\sum_{i} u_{i} v_{i}=0$ in $A$ then $\sum_{i} u_{i} x v_{i}=0$. So, $A$ is a nice SLS-algebra which is not Novikov.

Example 4. Suppose $V$ is a 3 -dimensional space with a basis $x, y, z$. Let $A$ be the nice SLS-algebra from Example 3, and let $A^{\prime}=A /(x z)$. Then $x y z \neq 0$ in $A^{\prime}$, so $A^{\prime}$ is an SLS-algebra which is not nice. 
Example 4 shows us that the class of nice SLS-algebra is not a variety: it is not closed relative to homomorphic images.

The main purpose of this section is to prove the following criterion.

Theorem 3. A left-symmetric algebra $(A, \circ)$ embeds into a differential Permalgebra in such a way that $a \circ b=a d(b)$ for $a, b \in A$ if and only if $A$ is a nice SLS-algebra.

The "only if" part is easy: the defining identities of an SLS-algebra holds on a differential Perm-algebra, and the map $\mu_{x}$ acts as a (left) multiplication by $d(x)$ :

for $a, b, x \in A$.

$$
\mu_{x}(a \circ b)=d(x) a d(b)=a d(x) d(b)=(a \circ x) \circ b
$$

To prove the "if" part, we first construct the universal enveloping Novikov dialgebra for a nice SLS-algebra and then apply Corollary 1 .

Suppose $(A, \circ)$ is a nice SLS-algebra. As above, let $T(A)$ stand for the (associative) tensor algebra of the space $A$ (without an identity), and let $\mathcal{D}(A)$ be the left-symmetric dialgebra constructed on $T(A)$ relative to the operations defined by (3.1), (3.2).

Let $V$ stand for the right ideal of the algebra $T(A)$ generated by

$$
g(a, x)=a x-\mu_{x}(a), \quad a \in A \circ A \subseteq A, x \in A .
$$

Choose a linear basis $X$ of $A$ in the form $X=X_{0} \cup X_{1}$ where $X_{0}$ is a linear basis of $A \circ A$ and $X_{1}$ is a complement of $X_{0}$. It is easy to see that

$$
V=\operatorname{Span}\left\{a x_{1} \ldots x_{n}-\mu_{x_{n}} \ldots \mu_{x_{1}}(a) \mid a \in X_{0}, x_{i} \in X, i \geq 1\right\} .
$$

In particular, $V \cap A=0$ since every generator of $V$ as of a linear space contains a unique principal term.

Note that

$$
(a \circ b) f-M(a f b) \in V
$$

for all $a, b \in A, f \in T(A)$, by the definition of $M$.

Denote

$$
K=\{f \in T(A) \mid M(a f b)=0 \text { for all } a, b \in A\}
$$

For example, $x y-y x \in K$. Moreover, if $f \in K$ then for all $u, v \in T(A) \cup\{1\}$ we have $u f v \in K$. Indeed,

$$
M(a u f v b)=M\left(M(a u) f x_{1} \ldots x_{n} b\right)=M\left(M\left(M(a u) f x_{1}\right) x_{2} \ldots x_{n} b\right)=0
$$

for $v=x_{1} \ldots x_{n}, n \geq 0$. Here we used the obvious corollary of the definition of $M$ :

$$
M(u v)=M(M(u) v), \quad u, v \in T(A) .
$$

In particular, if $f \in K$ and $u, v \in T(A)$ then $M(u f v)=0$.

Note that

$$
h(x, y, z)=x y z+(x \circ z) \circ y-(x \circ y) z-(x \circ z) y \in K
$$


for all $x, y, z \in A$. Indeed, compute

$$
\begin{aligned}
& M(a h(x, y, z) b)=M(a x y z+a(x \circ z) \circ y-a(x \circ y) z-a(x \circ z) y) \circ b \\
= & (((a \circ x) \circ y) \circ z+a \circ((x \circ z) \circ y)-(a \circ(x \circ y)) \circ z-(a \circ(x \circ z)) \circ y) \circ b \\
= & (((a \circ x) \circ z) \circ y+a \circ((x \circ z) \circ y)-(a \circ z) \circ(x \circ y)-(a \circ(x \circ z)) \circ y) \circ b=0 \circ b=0 .
\end{aligned}
$$

Let $I \subset T(A)$ stand for the linear span of all $u f v, u \in T(A), v \in T(A) \cup\{1\}$; this is a proper subset of $K$. In particular, $u x y-u y x \in I$ for all $u \in T(A)$, $x, y \in A$. Hence, we may permute variables in $T(A)$ modulo $I$ leaving the first letter unchanged.

Lemma 3. The set $I$ is a two-sided ideal of $\mathcal{D}(A)$.

Proof. For all $a \in A, u, v \in T(A)$ we have

$$
a \vdash(u v) \equiv(a \vdash u) v+u(a \vdash v)-u a v \quad(\bmod I) .
$$

It is easy to prove by induction on the length of $v$.

Let $f \in K, u \in T(A), v \in T(A) \cup\{1\}$. If $v \neq 1$ then $M(u f v)=0$ and thus

$$
u f v \vdash T(A)+T(A) \dashv u f v=0 .
$$

Consider the case $v=1$. For every $a, b \in A$, left symmetry implies

$$
\begin{aligned}
M(a M(u f) b) & =(a \circ M(u f)) \circ b \\
& =(a \circ(M(u f) \circ b)+(M(u f) \circ a) \circ b-M(u f) \circ(a \circ b)=0
\end{aligned}
$$

since $M(u f) \circ x=M(u f x)=0$ for all $x \in A$. Hence, $M(u f) \in K$.

Therefore, (5.2) leads to

$$
u f \vdash w=M(u f) \vdash w \equiv-(n-1) w M(u f) \equiv 0 \quad(\bmod I)
$$

for every $w \in T(A)$ of length $n$.

Similarly,

$$
w \dashv u f=w M(u f) \in I
$$

for every $w \in T(A)$.

This is a straightforward corollary of the definition that $I \vdash T(A) \subseteq I$. It remains to show $T(A) \dashv I \subseteq I$. Choose $u f v \in I$ for $f \in K, w \in T(A)$, and apply (5.2) for $x=M(w)$ :

$$
w \vdash u f v \equiv(x \vdash u) f v+u(x \vdash f) v+u f(x \vdash v)-2 u x f v \quad(\bmod I)
$$

(for $v \neq 1$; if $v=1$ then the equation is analogous but simpler). Obviously, we need to prove $x \vdash f \in K$ since all other summands belong to $I$. Indeed, consider

$$
x \vdash a f \equiv(x \circ a) f+a(x \vdash f)-a x f \quad(\bmod I) .
$$


Since $M(I) \circ b=0$, we obtain

$$
\begin{aligned}
M(a(x \vdash f) b)=M((x \vdash a f) b)-M((x \circ a) f b)+M(a x f b) & \\
& =M(x \vdash a f) \circ b=(x \circ M(a f)) \circ b=0
\end{aligned}
$$

due to left symmetry. Hence, $T(A) \vdash I \subseteq I$.

Lemma 4. The set $J=I+V$ is a two-sided ideal of the dialgebra $\mathcal{D}(A)$.

Proof. Since $I$ is already known to be an ideal, it remains to consider the right ideal $V$ of $T(A)$. By definition, $V \dashv T(A) \subseteq V$.

Denote

$$
g(a \circ b, x)=(a \circ b) x-(a \circ x) \circ b, \quad a, b, x \in A .
$$

Then $M(g(a \circ b, x) v)=0$ for all $v \in T(A)$ by (4.2), and thus $V A \vdash T(A)=0$. For $v=1$, we may see $M(g(a \circ b, x))=(a \circ b) \circ x-(a \circ x) \circ b$. Hence, $u M(g(a \circ b, x))=u((a \circ b) \circ x-(a \circ x) \circ b) \equiv u h(a, x, b)-u h(a, b, x) \equiv 0$ $(\bmod I)$. It follows that

$$
V \vdash T(A) \subseteq I
$$

by (5.2).

Similarly, $u \dashv g(a \circ b, x) v=u M(g(a \circ b, x) v) \in I$. for all $u \in T(A)$, $v \in T(A) \cup\{1\}$.

To complete the proof it remains to calculate

$$
\begin{aligned}
& c \vdash g(a \circ b, x)=c \vdash(a \circ b) x-c \circ((a \circ x) \circ b) \\
& =(c \circ(a \circ b)) x+(a \circ b)(c \circ x)-(a \circ b) c x-c \circ((a \circ x) \circ b) \\
& \equiv(c \circ x) \circ(a \circ b)+(a \circ(c \circ x)) \circ b-((a \circ c) \circ x) \circ b-c \circ((a \circ x) \circ b) \quad(\bmod V) .
\end{aligned}
$$

The latter relation is zero in every SLS-algebra. Then $u \vdash g(a \circ b, x) v \in J$ for all $u, v \in T(A)$ by (5.2).

Lemma 5. For all $u, w, v \in T(A)$ we have

$$
\begin{aligned}
& (u \dashv v) \dashv w-(u \dashv w) \dashv v \in J, \\
& (u \vdash v) \vdash w-(u \vdash w) \dashv v \in J .
\end{aligned}
$$

In particular, $\mathcal{D}(A) / J$ is a Novikov dialgebra.

Proof. The first relation follows from the definition of operations:

$$
(u \dashv v) \dashv w-(u \dashv w) \dashv v=u M(v) M(w)-u M(w) M(v) \in I .
$$

Let us prove the second one by induction on the length of $w$. For $w=a \in A$,

$$
\begin{gathered}
(u \vdash v) \vdash a=M(u \vdash v) \circ a=(M(u) \circ M(v)) \circ a, \\
(u \vdash a) \dashv v=(M(u) \circ a) M(v) \equiv \mu_{M(v)}(M(u) \circ a) \quad(\bmod V) .
\end{gathered}
$$


Hence, $(u \vdash v) \vdash a-(u \vdash a) \dashv v \in V \subset J$. Next,

$$
\begin{aligned}
& (u \vdash v) \vdash w a=((u \vdash v) \vdash w) a+w(M(u \vdash v) \circ a)-w M(u \vdash v) a \\
& \quad=((u \vdash v) \vdash w) a+w((M(u) \circ M(v)) \circ a)-w(M(u) \circ M(v)) a, \\
& (u \vdash w a) \dashv v=(u \vdash w a) M(v) \\
& \quad=(u \vdash w) a M(v)+w(M(u) \circ a) M(v)-w M(u) a M(v) \\
& \equiv(u \vdash w) M(v) a+w(M(u) \circ a) M(v)-w M(u) a M(v) \quad(\bmod I) .
\end{aligned}
$$

Hence,

$$
\begin{aligned}
&(u \vdash v) \vdash w a-(u \vdash w a) \dashv v \\
& \equiv((u \vdash v) \vdash w) a-(u \vdash w) M(v) a+w h(M(u), a, M(v)) \\
&=((u \vdash v) \vdash w-(u \vdash w) \dashv v) a+w h(M(u), a, M(v)) \equiv 0 \quad(\bmod J) .
\end{aligned}
$$

Remark 1. Suppose $A$ is a Novikov algebra. If $T$ is an ideal of the leftsymmetric algebra $\mathcal{D}(A)$ such that $\mathcal{D}(A) / T$ is a Novikov algebra then $T$ contains $u h(x, y, z)$ for all $u \in T(A), x, y, z \in A$ (it follows from the proof of Lemma 5). Therefore, the universal enveloping Novikov dialgebra $\mathcal{N}(A)$ of $A$ is actually an image of $A \oplus A^{\otimes 2} \otimes A^{\otimes 3} \subset T(A)$. In particular, if $A$ is finite-dimensional then so is $\mathcal{N}(A)$.

Lemma 6. $A \cap J=\{0\}$.

Proof. Recall that a linear basis $X$ of $A$ is chosen in the form $X=X_{0} \cup X_{1}$ where $X_{0}$ is a basis of $A \circ A$ and $X_{1}$ is its complement. Then $T(A)$ is the space of non-commutative polynomials in $X$.

By definition, the space $V$ is spanned by

$$
g\left(a, x_{1} \ldots x_{n}\right)=a x_{1} \ldots x_{n}-\mu_{x_{n}} \ldots \mu_{x_{1}}(a), \quad a \in X_{0}, x_{1}, \ldots, x_{n} \in X .
$$

Suppose $u \in T(A)$ is a word starting with a letter from $X_{0}: u=(a \circ b) w$. Then for every $f \in K, v \in T(A) \cup\{1\}$ we have

$$
u f v=(a \circ b) w f v \equiv M(a w f v b)=0 \quad(\bmod V)
$$

by (5.1).

Assume $J=I+V$ has a nonzero intersection with $A$. Then we may write the following equation in $T(A)$ :

$$
f_{1}+f_{0}+g=a \in A, \quad a \neq 0,
$$

where $g \in V, f_{i}$ is a linear combination of the elements like $u f v$ with $u$ starting with a letter from $X_{i}, i=0,1$. As we noted above, $f_{0} \in V$. Every monomial in $f_{1}$ is of length at least 2 by the definition of $I$. All monomials that emerge in elements of $V$ start with a letter from $X_{0}$, so $f_{1}$ must be zero. 
Therefore, we come to $a=f_{0}+g \in V \cap A$ which is impossible for $a \neq 0$.

Corollary 3. A nice SLS-algebra $(A, \circ)$ may be embedded into a Novikov dialgebra $(\mathcal{N}(A), \vdash,-)$ in such a way that $a \vdash b=a \circ b$ for $a, b \in A$.

Indeed, $\mathcal{N}(A)=\mathcal{D}(A) / J$ is the desired dialgebra.

Remark 2. Note that the generators of $I$ and $V$ represent the necessary conditions on a Novikov dialgebra which contains $(A, 0)$ in such a way that $a \vdash b=a \circ b$ for $a, b \in A$. Hence, $\mathcal{D}(A) / J$ is the universal enveloping Novikov dialgebra of $(A, \circ)$.

Now we may finish the proof of Theorem 3. A nice SLS-algebra $(A, \circ)$ embeds into a Novikov dialgebra $(\mathcal{N}(A), \vdash,-1)$, and the latter embeds into a differential Perm-algebra $(P, d)$ by Theorem 1. Since $a \circ b=a \vdash b=a d(b) \in P$ for $a, b \in A$, this is a desired embedding.

Corollary 4. The free SLS-algebra $\operatorname{SLS}\langle X\rangle$ is nice.

Corollary 5. The variety SLS is the smallest one containing the class of all special left-symmetric algebras.

\section{REFERENCES}

[1] B. Bakalov, A. D'Andrea, V. G. Kac, Theory of finite pseudoalgebras, Adv. Math. 162, 1-140 (2001).

[2] A. A. Balinskii, S. P. Novikov, Poisson brackets of hydrodynamic type, Frobenius algebras and Lie algebras, Sov. Math. Dokl., 32, 228-231 (1985).

[3] L. A. Bokut, Y. Chen, Z. Zhang, Gröbner-Shirshov bases method for GelfandDorfman-Novikov algebras, J. Algebra Appl., 16(1), 1750001, 22 pp. (2017).

[4] F. Chapoton. Un endofoncteur de la catégorie des opérades, In: Loday J.-L. , Frabetti A., Chapoton F., Goichot F. (Eds), Dialgebras and related operads, Springer-Verl., Berlin, 2001, pp. 105-110. (Lectures Notes in Math., vol. 1763).

[5] A. S. Dzhumadil'daev, C. Löfwall, Trees, free right-symmetric algebras, free Novikov algebras and identities, Homology, Homotopy Appl., 4 (2), 165-190 (2002).

[6] I. M. Gelfand, I. Ya. Dorfman, Hamilton operators and associated algebraic structures, Functional analysis and its application, 13, no. 4, 13-30 (1979).

[7] M. Gerstenhaber, The cohomology structure of an associative ring, Ann. Math., 78, 267-288 (1963).

[8] V. Yu. Gubarev, P. S. Kolesnikov. Embedding of dendriform algebras into Rota-Baxter algebras, Central European Journal of Mathematics, 11(2), 226-245 (2013).

[9] V. Yu. Gubarev, P. S. Kolesnikov. Operads of decorated trees and their duals, Comment. Math. Univ. Carolin., 55(4), 421-445 (2014).

[10] P. S. Kolesnikov, Varieties of dialgebras and conformal algebras, Sib. Math. Jour. 49(2), 257-272 (2008).

[11] P. S. Kolesnikov, B. Sartayev, A. Orazgaliev, Gelfand-Dorfman algebras, derived identities, and the Manin product of operads, Journal of Algebra 539, 260-284 (2019).

[12] P.S. Kolesnikov, B. K. Sartayev, On the special identities of Gelfand-Dorfman algebras, arXiv:2105.13815. 
[13] J.-L. Koszul, Domaines bornés homogènes et orbites de groupes de transformations affines, Bull. Soc. Math. Fr., 89, 515-533 (1961).

[14] D. Liu, Y. Pei, L.-M. Xia, On finite dimensional simple Novikov superalgebras. Comm. Algebra 47 (2019), no. 3, 999-1004.

[15] J.-L. Loday, Dialgebras, In: Loday J.-L., Frabetti A., Chapoton F., Goichot F. (Eds), Dialgebras and related operads, Springer-Verl., Berlin, 2001, pp. 105-110. (Lectures Notes in Math., vol. 1763).

[16] B. Vallette, Manin products, Koszul duality, Loday algebras and Deligne conjecture, J. Reine Angew. Math., 620, 105-164 (2008).

[17] J. Pei, C. Bai, L. Guo, X. Ni, Replicators, Manin white product of binary operads and average operators, In: New Trends in Algebra and Combinatorics, Proceedings of the 3rd International Congress in Algebra and Combinatorics (K. P. Shum et al, Eds.), World Scientific, 255-267 (2020).

[18] A. Pozhidaev, 0-dialgebras with bar-unity, Rota-Baxter and 3-Leibniz algebras, in Groups, Rings and Group Rings, ed. A. Giambruno et al. (Providence, RI: American Mathematical Society (AMS), 2009) pp. 245-256.

[19] E. B. Vinberg, Homogeneous cones, Sov. Math. Dokl., 1, 787-790 (1960).

Sobolev Institute of Mathematics, Novosibirsk, Russia 